\title{
BENDING DEFORMATIONS OF COMPLEX HYPERBOLIC SURFACES
}

\author{
Boris Apanasov $\dagger$
}

\begin{abstract}
We study deformations of complex hyperbolic surfaces which furnish the simplest examples of : (i) negatively curved Kähler manifolds and (ii) negatively curved Riemannian manifolds not having constant curvature. Although such complex surfaces may share the rigidity of quaternionic/octionic hyperbolic manifolds, our main goal is to show that they enjoy nevertheless the flexibility of low-dimensional real hyperbolic manifolds. Namely we define a class of "bending" deformations of a given (Stein) complex surface $M$ associated with its closed geodesics provided that $M$ is homotopy equivalent to a Riemann surface whose embedding in $M$ has a nontrivial totally real geodesic part. Such bending deformations bend $M$ along its closed geodesics and are induced by equivariant quasiconformal homeomorphisms of the complex hyperbolic space and its Cauchy-Riemannian structure at infinity.
\end{abstract}

\section{INTRODUCTION}

A complex hyperbolic manifold $M$ is locally modeled on the complex hyperbolic space $\mathbb{H}_{\mathbb{C}}^{n}$ which can be represented by the unit complex ball $\mathbb{B}^{n} \subset \mathbb{C}^{n}$ with the Kähler structure given by the Bergman metric (with pinched negative curvature $K,-1 \leq K \leq-1 / 4$, compare [M2, GP, G3, AX1]). In particular, in complex dimension two, due to Yau's uniformization theorem [Ya], every smooth complex projective surface $M$ with positive canonical bundle satisfying the topological condition $\chi(M)=3 \cdot \operatorname{Signature}(M)$ is a complex hyperbolic manifold. Since $M=\mathbb{H}_{\mathbb{C}}^{n} / G$, where the discrete torsion free group $G \subset P U(n, 1)$ is the fundamental group of $M$, one can reduce the study of the Teichmüller space $\mathcal{T}(M)$ of isotopy classes of complex hyperbolic structures on $M$ to studying the variety $\mathcal{T}(G)$ of conjugacy classes of discrete faithful representations $\rho: G \rightarrow P U(n, 1)$ (involving the space $\mathcal{D}(M)$ of the developing maps, see [G2, FG]). Here $\mathcal{T}(G)=\mathcal{R}_{0}(G) / P U(n, 1)$, and the variety $\mathcal{R}_{0}(G) \subset \operatorname{Hom}(G, P U(n, 1))$ consists of discrete faithful representations $\rho$ of the group $G$ whose co-volume, $\operatorname{Vol}\left(\mathbb{H}_{\mathbb{C}}^{n} / G\right)$, may be infinite.

Due to the Mostow rigidity theorem [M1], hyperbolic structures of finite volume and (real) dimension at least three are uniquely determined by their topology, and one has no continuous deformations of them. Despite that, real hyperbolic manifolds $M$ can be deformed as conformal manifolds, or equivalently as higherdimensional hyperbolic manifolds $M \times(0,1)$ of infinite volume. First such deformations were given by the author [A2] and, after Thurston's "Mickey Mouse" example

1991 Mathematics Subject Classification. 57,55,53,51,30.

Key words and phrases. Complex hyperbolic manifolds; Cauchy-Riemannian structure; disk bundles over surfaces; quasiconformal mappings; varieties of representations; Teichmüller space; bending deformations. 
[Th], were called bendings of $M$ along its totally geodesic hypersurfaces, see also [A1, A3-A5, JM, Ko]. Furthermore all these deformations are quasiconformally equivalent showing a rich supply of quasiconformal $G$-equivariant homeomorphisms in the closure of the real hyperbolic space $\mathbb{H}_{\mathbb{R}}^{n}$. In particular the limit set $\Lambda(G) \subset$ $\partial \mathbb{H}_{\mathbb{R}}^{n+1}$ deforms continuously from a round sphere $\partial \mathbb{H}_{\mathbb{R}}^{n}=S^{n-1} \subset S^{n}=\mathbb{H}_{\mathbb{R}}^{n+1}$ into a nondifferentiably embedded topological $(n-1)$-sphere quasiconformally equivalent to $S^{n-1}$.

Contrasting to the above flexibility, "non-real" hyperbolic manifolds seem much more rigid. In particular, due to Pansu $[\mathrm{P}]$, quasiconformal maps in the sphere at infinity of quaternionic/octionic hyperbolic spaces are necessarily automorphisms, and thus there cannot be interesting quasiconformal deformations of corresponding structures. Secondly, due to Corlette's rigidity theorem [Co], such manifolds are even super-rigid - analogously to Margulis super-rigidity in higher rank [Ma]. Furthermore, complex hyperbolic manifolds share the above rigidity of quaternionic/octionic hyperbolic manifolds. Namely, due to the Goldman's local rigidity theorem in dimension $n=2$ [G1], every nearby discrete representation $\rho: G \rightarrow$ $P U(2,1)$ of a cocompact lattice $G \subset P U(1,1)$ stabilizes a complex geodesic in the complex hyperbolic space $\mathbb{H}_{\mathbb{C}}^{2}$ (which is also true for small deformations of cocompact lattices $G \subset P U(n-1,1)$ in higher dimensions $n \geq 3[\mathrm{GM}])$, and thus the limit set $\Lambda(\rho G) \subset \partial \mathbb{H}_{\mathbb{C}}^{n}$ is always a round sphere $S^{2 n-3}$. In higher dimensions $n \geq 3$, this local rigidity of complex hyperbolic $n$-manifolds $M$ homotopy equivalent to their closed complex totally geodesic hypersurfaces is even global due to a recent Yue's theorem $[\mathrm{Yu}]$.

Our goal here is to show that, in contrast to rigidity of complex hyperbolic nmanifolds $M$ from the above class, complex hyperbolic (Stein) manifolds $M$ homotopy equivalent to their closed totally real geodesic surfaces are not rigid. Namely, in complex dimension 2, we provide a canonical construction of continuous non-trivial quasi-Fuchsian deformations of complex surfaces fibered over closed Riemannian surfaces. This is the first such deformations of fibrations with compact base (for non-compact base, see a different Goldman-Parker' deformation [GP] of ideal triangle groups $G \subset P O(2,1))$. Our construction is inspired by the well know bending deformations of real hyperbolic (conformal) manifolds along totally geodesic hypersurfaces and by a M.Carneiro-N.Gusevskii' construction of a discrete representation in $P U(2,1)$ of a surface group, which has been kindly showed us by N.Gusevskii $[\mathrm{Gu}]$. In the case of complex hyperbolic (and Cauchy-Riemannian) structures, it works however in a different way than in the real one. Namely our complex bending deformations involve simultaneous bending of the base of the fibration of the complex surface $M$ as well as bendings of each of its totally geodesic fibers (see Remark 4.10). Such bending deformations of complex surfaces are associated to their real simple closed geodesics (of real codimension 3), but have nothing common with cone deformations of real hyperbolic 3-manifolds along closed geodesics (see $[\mathrm{A} 4, \mathrm{~A} 5])$.

We also remark that the condition that the group $G \subset P U(n, 1)$ preserves a complex totally geodesic hyperspace in $\mathbb{H}_{\mathbb{C}}^{n}$ is essential for local rigidity of deformations only for co-compact lattices $G \subset P U(n-1,1)$. As our subsequent work [ACG] shows, there are non-trivial quasi-Fuchsian deformations of such a co-finite lattice $G \subset P U(1,1)$ in $P U(2,1)$ induced by a continuous family of $G$-equivariant 
obstruction.

Furthermore, there are well known complications (cf. [KR, P, V]) in constructing equivariant homeomorphisms in the complex hyperbolic space and in CauchyRiemannian geometry, which are due to necessary conditions for such maps to preserve the Kähler and contact structures (correspondingly in the complex hyperbolic space and at its infinity, the one-point compactification of the Heisenberg group $\left.\mathcal{H}_{n}\right)$. Despite that, as it follows from our construction, the complex bending deformations are induced by equivariant homeomorphisms which are in addition quasiconformal with respect to the corresponding metrics. One of our main results may be formulated as follows.

Theorem 4.7. Let $G \subset P O(2,1) \subset P U(2,1)$ be a given lattice uniformizing a Riemann 2-surface $S_{p}$ of genus $p \geq 2$. Then, for any simple closed geodesic $\alpha \subset S_{p}=H_{\mathbb{R}}^{2} / G$ and a sufficiently small $\eta_{0}>0$, there is a bending deformation $\mathcal{B}_{\alpha}:\left(-\eta_{0}, \eta_{0}\right) \rightarrow \mathcal{R}_{0}(G)$ of the group $G$ along $\alpha, \mathcal{B}_{\alpha}(\eta)=\rho_{\eta}=F_{\eta}^{*}$, induced by G-equivariant quasiconformal homeomorphisms $F_{\eta}: \overline{\mathbb{H}_{\mathbb{C}}^{2}} \rightarrow \overline{\mathbb{H}_{\mathbb{C}}^{2}}$.

We notice that such complex bending deformations depend on many independent parameters, as it is shown by application of our construction and Élie Cartan [Ca] angular invariant in Cauchy-Riemannian geometry:

Corollary 4.8. Let $S_{p}=\mathbb{H}_{\mathbb{R}}^{2} / G$ be a closed totally real geodesic surface of genus $p>1$ in a given complex hyperbolic surface $M=\mathbb{H}_{\mathbb{C}}^{2} / G, G \subset P O(2,1) \subset P U(2,1)$. Then there is an embedding $\pi \circ \mathcal{B}: B^{2 p-2} \hookrightarrow \mathcal{T}(M)$ of a real $(2 p-2)$-ball into the Teichmüller space of $M$, defined by bending deformations along disjoint closed geodesics in $M$ and the projection $\pi: \mathcal{D}(M) \rightarrow \mathcal{T}(M)=\mathcal{D}(M) / P U(2,1)$.

In our subsequent work $[\mathrm{AG}]$, we apply the constructed bending deformations to answer a well known question about cusp groups on the boundary of the Teichmüller space of $\mathcal{T}(M)$ of a Stein complex surface $M$ fibering over a compact Riemann surface of genus $p>1$ :

Theorem. Let $G \subset P O(2,1) \subset P U(2,1)$ be a uniform lattice isomorphic to the fundamental group of a closed surface $S_{p}$ of genus $p \geq 2$. Then, for any simple closed geodesic $\alpha \subset S_{p}=H_{\mathbb{R}}^{2} / G$, there is a continuous deformation $\rho_{t}=f_{t}^{*}$ induced by G-equivariant quasiconformal homeomorphisms $f_{t}: \overline{\mathbb{H}_{\mathbb{C}}^{2}} \rightarrow \overline{\mathbb{H}_{\mathbb{C}}^{2}}$ whose limit representation $\rho_{\infty}$ corresponds to a boundary cusp point of the Teichmüller space $\mathcal{T}(G)$, that is the boundary group $\rho_{\infty}(G)$ has an accidental parabolic element $\rho_{\infty}\left(g_{\alpha}\right)$ where $g_{\alpha} \in G$ represents the geodesic $\alpha \subset S_{p}$.

We note that, due to our construction of such continuous quasiconformal deformations in $[\mathrm{AG}]$, they are independent if the corresponding geodesics $\alpha_{i} \subset S_{p}$ are disjoint. It implies the existence of a boundary group in $\partial \mathcal{T}(G)$ with "maximal" number of non-conjugate accidental parabolic subgroups:

Corollary. Let $G \subset P O(2,1) \subset P U(2,1)$ be a uniform lattice isomorphic to the fundamental group of a closed surface $S_{p}$ of genus $p \geq 2$. Then there is a continuous deformation $R: \mathbb{R}^{2 p-2} \rightarrow \mathcal{T}(G)$ whose boundary group $G_{\infty}=R(\infty)(G)$ has $2 p-2$ non-conjugate accidental parabolic subgroups.

Finally, we mention another aspect of the intrigue problem on geometrical finiteness of complex hyperbolic surfaces (see [AX1, AX2]) for which it may be possible 
Problem. Construct a geometrically infinite (finitely generated) discrete group $G \subset P U(2,1)$ whose limit set is the whole sphere at infinity, $\Lambda(G)=\partial \mathbb{H}_{\mathbb{C}}^{2}=\overline{\mathcal{H}}$, and which is the limit of convex cocompact groups $G_{i} \subset P U(2,1)$ from the Teichmüller space $\mathcal{T}(\Gamma)$ of a convex cocompact group $\Gamma \subset P U(2,1)$. Is that possible for a Schottky group $\Gamma$ ?

Acknowledgements. The author would like to thank Nikolay Gusevskii for helpful conversations. A part of paper was written during the author's stay at MSRI. Research at MSRI was supported in part by NSF grant DMS-9022140.

\section{COMPleX HyPerbolic SURFACES HOMOTOPY EQUIVALENT TO A RIEMANN SURFACE}

Let $M$ be a complex hyperbolic surface (a complete Kähler 2-manifold of constant holomorphic sectional curvature -1) homotopy equivalent to a Riemann surface $S_{g}$ of genus $g>1$, that is $M=\mathbb{H}_{\mathbb{C}}^{2} / G$ is a quotient of the complex hyperbolic 2space $\mathbb{H}_{\mathbb{C}}^{2}$ by a discrete torsion free isometry group $G \subset P U(2,1)$ isomorphic to the fundamental group $\pi_{1}\left(S_{g}\right)$. The boundary at infinity of $M$ is a 3-dimensional manifold with the induced spherical Cauchy-Riemannian structure modeled on the one-point compactification $\overline{\mathcal{H}}=\mathcal{H} \cup\{\infty\} \approx S^{3}$ of the Heisenberg group $\mathcal{H}=\mathbb{C} \times \mathbb{R}$.

We are interested in Teichmüller spaces of such complex surfaces and CauchyRiemannian 3-manifolds or equivalently, in varieties of conjugacy classes of discrete faithful representations $\rho: G \rightarrow P U(2,1)$, especially in curves in these spaces corresponding to continuous families of quasiconformal deformations of such structures or discrete groups.

As the simplest examples of such complex surfaces, one can take the quotients $M_{i}=\mathbb{H}_{\mathbb{C}}^{2} / G_{i}$ corresponding to two embeddings of $\pi_{1}\left(S_{g}\right)$ as lattices acting on totally geodesic planes in $\mathbb{H}_{\mathbb{C}}^{2}$, either on complex geodesics (represented by $\mathbb{H}_{\mathbb{C}}^{1} \subset \mathbb{H}_{\mathbb{C}}^{2}$ ) or on totally real geodesic 2-planes (represented by $\mathbb{H}_{\mathbb{R}}^{2} \subset \mathbb{H}_{\mathbb{C}}^{2}$ ). In these cases the discrete surface group is either $G_{1} \subset P U(1,1) \subset P U(2,1)$ or $G_{2} \subset P O(2,1) \subset$ $P U(2,1)$, correspondingly. The limit sets $\Lambda\left(G_{1}\right)$ and $\Lambda\left(G_{2}\right)$ are homeomorphic to the circle $S^{1}$. Moreover, the actions of the groups $G_{1}$ and $G_{2}$ on such circles are (equivariantly) homeomorphically conjugate due to the following Apanasov [A6] isomorphism theorem:

Theorem 2.1. Let $\varphi: G \rightarrow H$ be a type preserving isomorphism of two non-elementary geometrically finite discrete subgroups $G, H \subset P U(n, 1)$. Then there exists a unique equivariant homeomorphism $f_{\varphi}: \Lambda(G) \rightarrow \Lambda(H)$ of their limit sets that induces the isomorphism $\varphi$.

However, in the case of the above surface groups $G_{1}$ and $G_{2}$, the equivariant homeomorphism $f_{\varphi}: \Lambda\left(G_{1}\right) \rightarrow \Lambda\left(G_{2}\right)$ cannot be homeomorphically extended to the whole sphere $\partial \mathbb{H}_{\mathbb{C}}^{2} \approx S^{3}$. The obstruction to that is due to the fact that the quotient complex surfaces $\mathbb{H}_{\mathbb{C}}^{2} / G_{1}$ and $\mathbb{H}_{\mathbb{C}}^{2} / G_{2}$ are not homeomorphic. Namely, these complex surfaces are disc bundles over $S_{g}$ and have different Toledo invariants: $\tau\left(\mathbb{H}_{\mathbb{C}}^{2} / G\right)=2 g-2$ and $\tau\left(\mathbb{H}_{\mathbb{C}}^{2} / H\right)=0$, see $[\mathrm{To}]$.

The complex structures of the complex surfaces $M_{1}$ and $M_{2}$ are quite different, too. While the manifolds $M_{1}$ (corresponding to $G_{1} \subset P U(1,1)$ ) have natural embeddings of the Riemann surface $S_{g}$ as holomorphic totally geodesic submanifolds and hence cannot be Stein manifolds if $S_{p} \subset M_{1}$ is compact, the manifolds $M_{2}$ 
Moreover due to Goldman [G1], if the surface $S_{p} \subset M_{1}$ is closed (i.e. the lattices $G_{1} \subset P U(1,1)$ are co-compact), the manifolds $M_{1}$ are locally rigid in the sense that every nearby representation $G_{1} \rightarrow P U(2,1)$ stabilizes a complex geodesic in $\mathbb{H}_{\mathbb{C}}^{2}$ and is conjugate to a representation $G_{1} \rightarrow P U(1,1) \subset P U(2,1)$. In other words, there are no non-trivial "quasi-Fuchsian" deformations of $G_{1}$ and $M_{1}$.

Our goal here is to show that, in contrast to such rigidity of manifolds $M_{1}$ obtained from uniform lattices in $P U(1,1)$, the Stein manifolds $M_{2}$ obtained from discrete subgroups in $P O(2,1)$ are non-rigid. Furthermore, for a given simple geodesic $\alpha \subset M_{2}$ (in the totally real geodesic surface $S_{g} \subset M_{2}$ ), there is a continuous non-trivial path in the Teichmüller space $\mathcal{T}\left(M_{2}\right)$ (or $\mathcal{T}\left(G_{2}\right)$ ) represented by a continuous family of $G_{2}$-equivariant quasiconformal homeomorphisms $f_{\alpha, t}: \overline{\mathcal{H}} \rightarrow \overline{\mathcal{H}}$. We call such a deformation $\mathcal{B}_{\alpha}, \mathcal{B}_{\alpha}(t)=f_{\alpha, t}^{*}$, a bending deformation of CauchyRiemannian 3-manifold $\partial_{\infty} M_{2}$, the group $G_{2}$ and the complex hyperbolic surface $M_{2}$ along a given closed geodesic $\alpha \subset M_{2}$.

\section{Colar lemma And Dirichlet polyhedra}

For a given discrete group $\Gamma \subset P O(2,1) \subset P U(2,1)$, let $S=\mathbb{H}_{\mathbb{R}}^{2} / \Gamma$ be a real hyperbolic 2-orbifold embedded in $M=\mathbb{H}_{\mathbb{C}}^{2} / \Gamma$ as a totally real geodesic suborbifold. While $M$ has constant holomorphic sectional curvature -1, its sectional curvature varies in $[-1,-1 / 4]$, and in particular the real 2-orbifold $S$ has constant sectional curvature $K=-1 / 4$.

For a simple closed geodesic $\alpha \subset S \subset M$, let $\gamma_{\alpha} \in \pi_{1}^{\text {orb }}(S) \cong \Gamma$ represent it. Then the fundamental group $\pi_{1}^{\text {orb }}(S) \cong \Gamma$ can be decomposed into either a free amalgamated product or HNN-extension of its subgroups. Namely, for $\left.\Gamma_{0}=<\gamma_{\alpha}\right\rangle$, we correspondingly have for separating and non-separating geodesics $\alpha$ :

$$
\Gamma=\Gamma_{1} *_{\Gamma_{0}} \Gamma_{2} \quad \text { and } \quad \Gamma=\Gamma_{1} *_{\Gamma_{0}} .
$$

Here we would like to focus on an analogue of the well-known collar lemma which (for Riemann surfaces) is originally due to L.Keen [Ke] and its sharp form due to P.Buser [Bu]. It claims that a simple closed geodesic $\alpha$ of length $\ell$ on a Riemann surface $S$ of constant curvature -1 has a collar neighborhood $U_{\delta}(\alpha) \subset S$ of radius $\delta=\ln (\operatorname{coth} \ell / 4)$, that is no disjoint closed geodesic $\beta \subset S, \alpha \cap \beta=\emptyset$, intersects the neighborhood $U_{\delta}(\alpha)$.

To reformulate the collar lemma for isometric action of a discrete group $\Gamma \subset$ $P O(2,1) \subset P U(2,1)$ on the real hyperbolic plane $\mathbb{H}_{\mathbb{R}}^{2} \subset \mathbb{H}_{\mathbb{C}}^{2}$, we denote $A \subset \mathbb{H}_{\mathbb{R}}^{2}$ the axis of a hyperbolic element $\gamma_{\alpha} \in \Gamma$ (the translation along $A$ of the length $\ell$ ). Namely, multiplying lengths by $\sqrt{-K}$, we have due to Beardon [Be, 11.6.10] that, for any such discrete isometric $\Gamma$-action on the real hyperbolic plane of constant negative curvature $K<0$,

$$
\sinh \left(\frac{\ell \sqrt{-K}}{2}\right) \cdot \sinh \left(\frac{d(A, g(A)) \sqrt{-K}}{2}\right) \geq \frac{1}{2},
$$

for any $\gamma \in \Gamma \backslash \Gamma_{0}, \quad \Gamma_{0}=<\gamma_{\alpha}>$.

This shows that, for any element $\gamma \in \Gamma \backslash \Gamma_{0}$ of the surface group $\Gamma \subset P O(2,1) \subset$ $P U(2,1), \Gamma \cong \pi_{1}(S)$, which does not preserve the axis $A$ of the cyclic hyperbolic 


$$
\sinh \left(\frac{\ell}{4}\right) \cdot \sinh \left(\frac{d(A, g(A))}{4}\right) \geq \frac{1}{2} .
$$

Lemma 3.2. Let $\gamma_{\alpha}$ be a hyperbolic element of a non-elementary discrete group $\Gamma \subset P O(2,1) \subset P U(2,1)$ with translation length $\ell$ along its axis $A \subset \mathbb{H}_{\mathbb{R}}^{2}$, and $\delta>0$ a radius of a tubular neighborhood $U_{\delta}(A)$ precisely invariant with respect to its stabilizer $\Gamma_{0} \subset \Gamma$ :

$$
\Gamma_{0}\left(U_{\delta}(A)\right)=U_{\delta}(A) \quad \text { and } \quad \gamma\left(\left(U_{\delta}(A)\right) \cap U_{\delta}(A)=\emptyset, \quad \gamma \in \Gamma \backslash \Gamma_{0} .\right.
$$

Then the Dirichlet polyhedron $D_{z}(\Gamma)$ of the group $\Gamma$ centered at a point $z \in A$ has two sides $\sigma, \sigma^{\prime}, \gamma_{\alpha}(\sigma)=\sigma^{\prime}$, provided that $\delta>\ell / 4$.

Proof. Since the central point $z$ lies on the $\Gamma$-invariant totally real geodesic plane $\mathbb{H}_{\mathbb{R}}^{2} \subset \mathbb{H}_{\mathbb{C}}^{2}$, the Dirichlet polyhedron $D_{z}(\Gamma)$ of the group $\Gamma$ has the same combinatorics as its restriction to $\mathbb{H}_{\mathbb{R}}^{2}$ (the Dirichlet polygon there), see [G3, VIII.4]. On the plane $\mathbb{H}_{\mathbb{R}}^{2}$, the worst case is the case of an element $\gamma \in \Gamma \backslash \Gamma_{0}$ that maps the point $z$ to a point $\gamma(z)$ which lies in the intersection of one of Dirichlet bisectors $\mathfrak{S}\left(z, \gamma_{\alpha}(z)\right)$ and $\mathfrak{S}\left(z, \gamma_{\alpha}^{-1}(z)\right)$,

$$
\mathfrak{S}\left(z_{1}, z_{2}\right)=\left\{w \in \mathbb{H}_{\mathbb{C}}^{2}: d\left(z_{1}, w\right)=d\left(z_{2}, w\right)\right\}
$$

and the boundary $\partial U_{2 \delta}(A)$ of the $2 \delta$-neighborhood of the axis $A$. Considering the right geodesic triangle with vertices $z, \gamma(z)$ and $A \cap \mathfrak{S}\left(z, \gamma_{\alpha}(z)\right)$, we clearly see that the bisector $\mathfrak{S}(z, \gamma(z))$ passes the point $A \cap \mathfrak{S}\left(z, \gamma_{\alpha}(z)\right)$ if and only if $\ell=4 \delta$. This completes the proof.

Corollary 3.3. Let $\gamma_{\alpha}$ be a hyperbolic element of a non-elementary discrete group $\Gamma \subset P O(2,1) \subset P U(2,1)$ with translation length $\ell$ along its axis $A \subset \mathbb{H}_{\mathbb{R}}^{2}$. Then any tubular neighborhood $U_{\delta}(A)$ of the axis $A$ of radius $\delta>0$ is precisely invariant with respect to its stabilizer $\Gamma_{0} \subset \Gamma$ if

$$
\sinh \left(\frac{\ell}{4}\right) \cdot \sinh \left(\frac{\delta}{2}\right) \leq \frac{1}{2} .
$$

Furthermore, for sufficiently small $\ell, \ell<4 \delta$, the Dirichlet polyhedron $D_{z}(\Gamma)$ of the group $\Gamma$ centered at a point $z \in A$ has two sides $\sigma$ and $\sigma^{\prime}$ intersecting the axis $A$ and such that $\gamma_{\alpha}(\sigma)=\sigma^{\prime}$.

Remark 3.5. It is easy to see that both conditions of Corollary hold for $\ell>0$ and $\delta>0$ such that

$$
\begin{aligned}
\sinh \frac{\ell}{2} & <\frac{1}{2} \operatorname{csch}\left(\frac{\ln 63}{4}\right) \approx 0.406114 \\
\ln 63 & \leq \delta<\operatorname{arccsch}\left(2 \sinh \frac{\ell}{4}\right)
\end{aligned}
$$

The first condition in particular holds for $\ell<0.791411$ (see also [GKL]).

Proof. The claim about the sides of the Dirichlet polyhedron $D_{z}(\Gamma)$ directly follows from the Lemma 3.2. In particular, it is easy to verify that the values of the length 
To show that $U_{\delta}(A)$ is precisely $\Gamma_{0}$-invariant in $\mathbb{H}_{\mathbb{C}}^{2}$ it is enough to prove this fact for the restriction of $\Gamma$-action to the plane $\mathbb{H}_{\mathbb{R}}^{2}$ (see also [GKL]). Here, due to (3.1) and the hypothesis (3.4), we have:

$$
\sinh \frac{\delta}{2} \leq \frac{1}{2} \operatorname{csch}\left(\frac{\ell}{4}\right) \leq \sinh \left(\frac{d(A, g(A))}{4}\right) .
$$

This shows that

$$
\sinh \frac{\delta}{2}<\sinh \frac{d(A, \gamma(A))}{4} \quad \text { for any } \quad \gamma \in \Gamma \backslash \Gamma_{0},
$$

which implies that $d(A, \gamma(A))>2 \delta$, and hence $\gamma\left(\left(U_{\delta}(A)\right) \cap U_{\delta}(A)=\emptyset\right.$ for any $\gamma \in \Gamma \backslash \Gamma_{0}$.

\section{Bendings of COMPleX SuRfaCes AlONG Simple ClOSED GeOdesics}

Now we start with a totally real geodesic surface $S=\mathbb{H}_{\mathbb{R}}^{2} / \Gamma$ in the complex surface $M=\mathbb{H}_{\mathbb{C}}^{2} / \Gamma$, where $\Gamma \subset P O(2,1) \subset P U(2,1)$ is a given discrete group, and fix a simple geodesic $\alpha$ on $S$. According to the previous sections 2 and 3, we may assume that the loop $\alpha$ is covered by a geodesic $A \subset \mathbb{H}_{\mathbb{R}}^{2} \subset \mathbb{H}_{\mathbb{C}}^{2}$ whose ends at infinity are $\infty$ and the origin of the Heisenberg group $\mathcal{H}=\mathbb{C} \times \mathbb{R}, \overline{\mathcal{H}}=\partial \mathbb{H}_{\mathbb{C}}^{2}$. Furthermore, deforming the surface $S$ and its holonomy group $\Gamma \subset P O(2,1)$ (bending along the geodesic $\alpha$ ), we can assume that the hyperbolic length of $\alpha$ is sufficiently small and the radius of its tubular neighborhood is big enough - as in Corollary 3.3 and Remark 3.5.

Then the group $G$ and its subgroups $G_{0}, G_{1}, G_{2}$ in the free amalgamated (or HNN-extension) decomposition of $G$ have Dirichlet polyhedra $D_{z}\left(G_{i}\right) \subset \mathbb{H}_{\mathbb{C}}^{2}, i=$ $0,1,2$, centered at a point $z \in A=(0, \infty)$, whose intersections with the hyperbolic 2-plane $\mathbb{H}_{\mathbb{R}}^{2}$ have the shapes indicated in Figures 1-4.

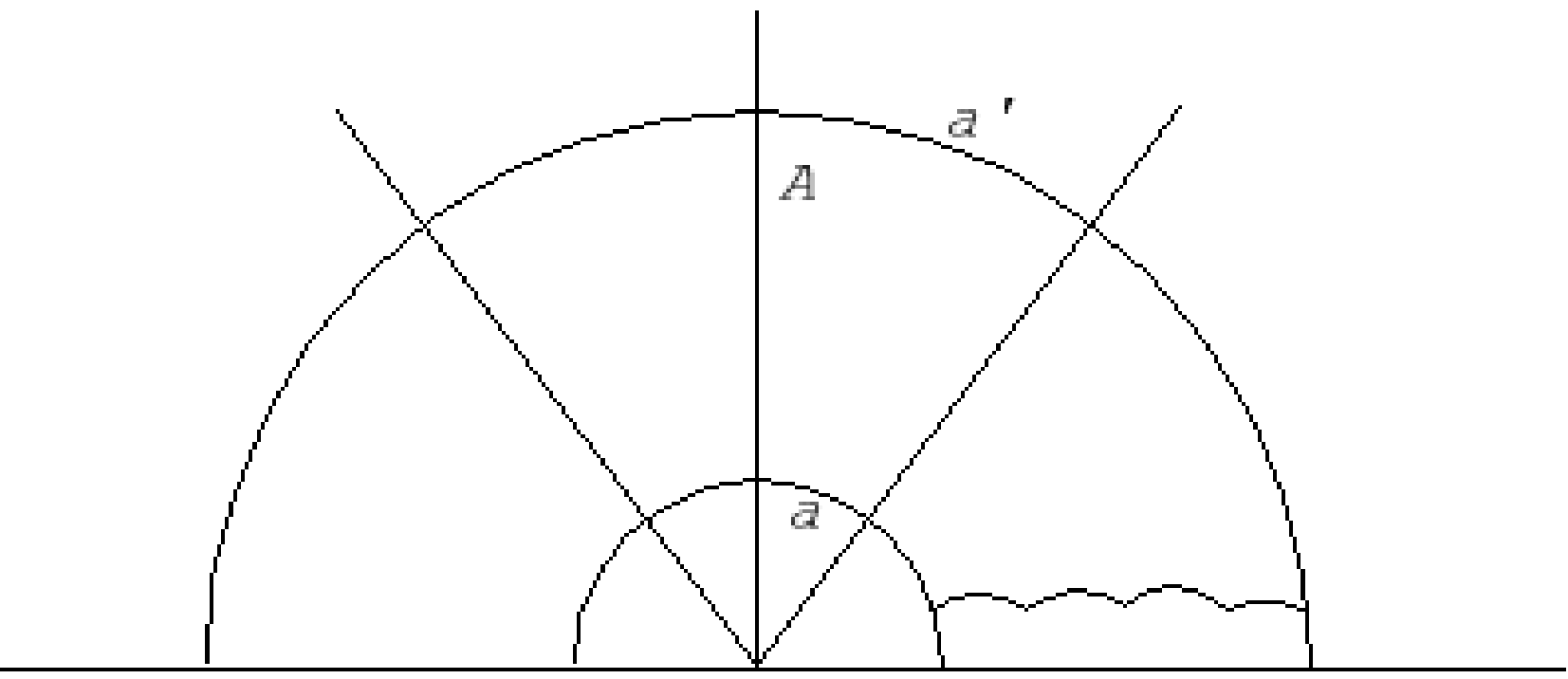




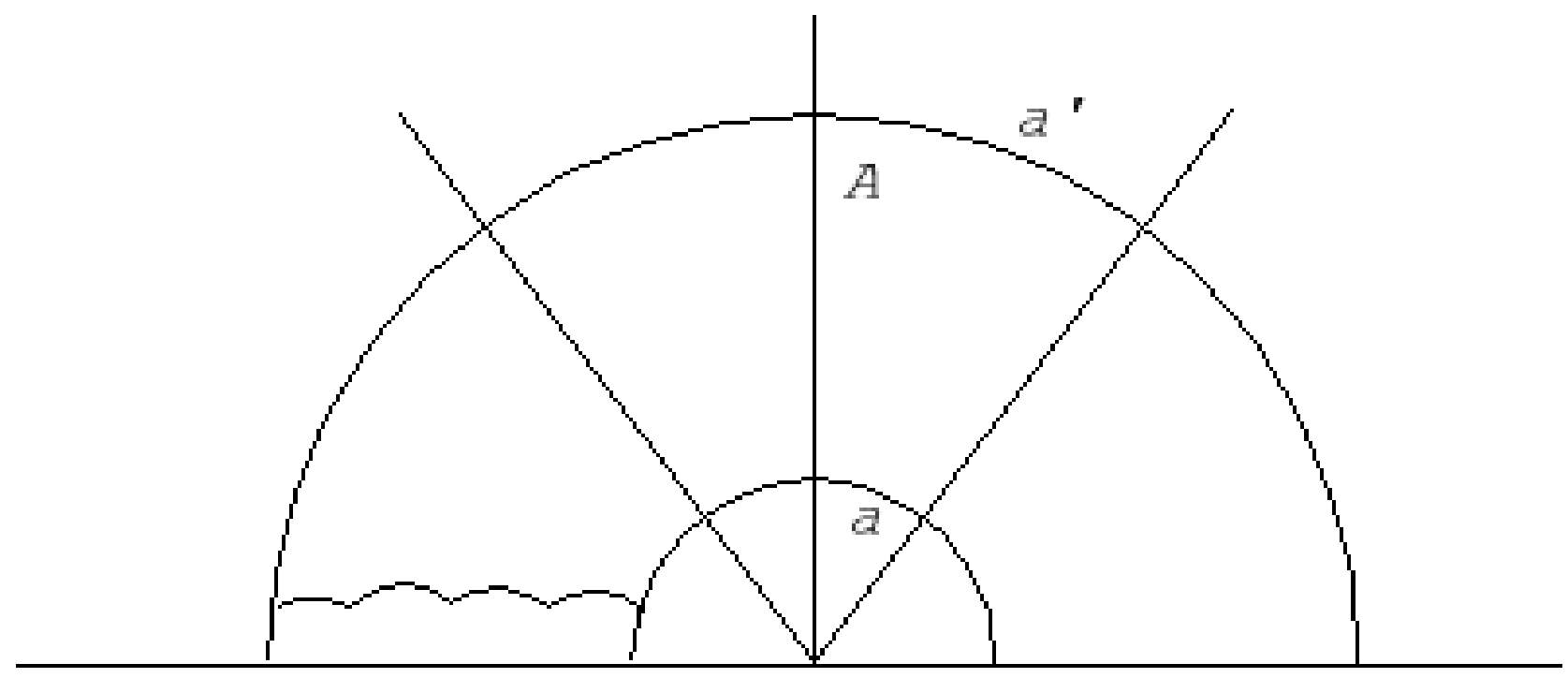

Figure 2. $G_{2} \subset G=G_{1} *_{G_{0}} G_{2}$.

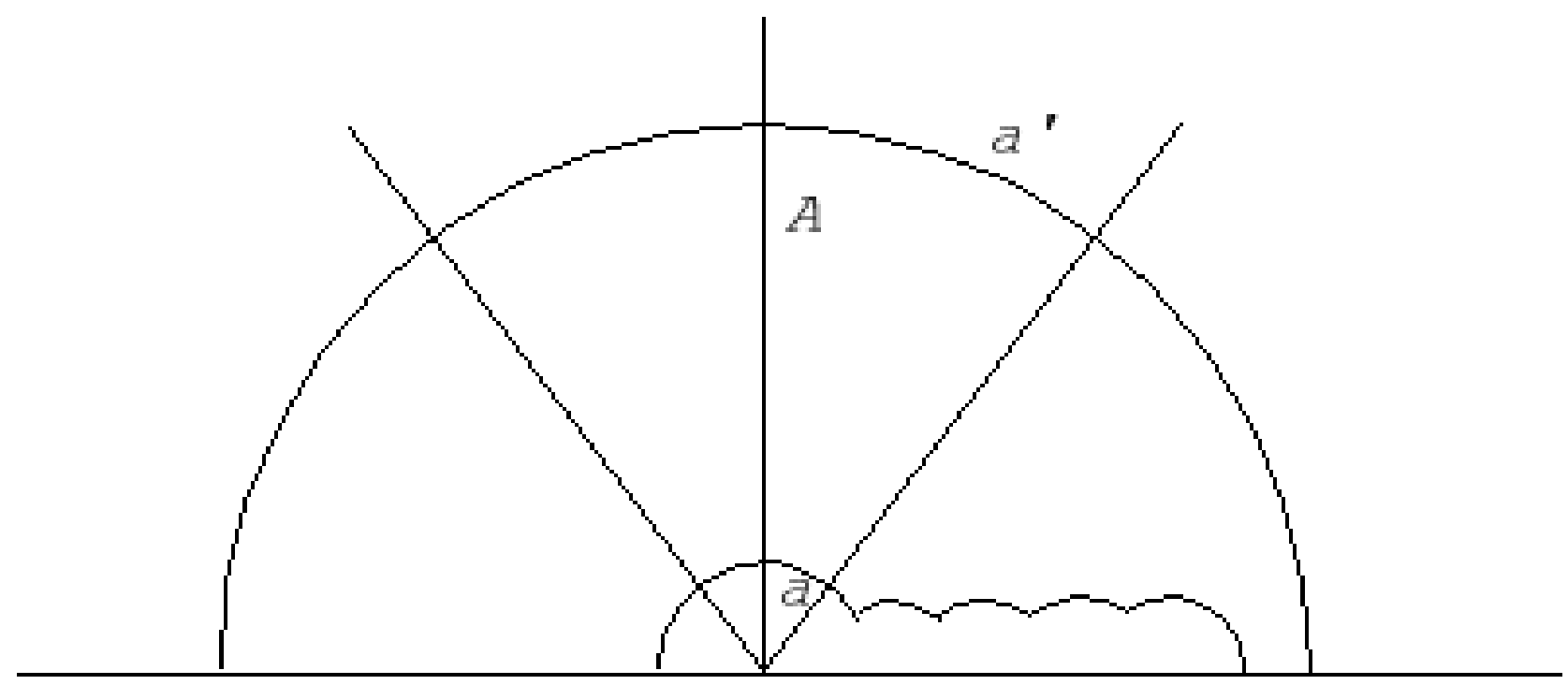

Figure 3. $G_{1} \subset G=G_{1} *_{G_{0}}$.

In particular we have that, except two bisectors $\mathfrak{S}$ and $\mathfrak{S}^{\prime}$ that are equivalent under the hyperbolic translation $g_{\alpha}$ (which generates the stabilizer $G_{0}$ of the axis $A$ ), all other bisectors bounding those Dirichlet polyhedra lie in sufficiently small "cone neighborhoods" $C_{+}$and $C_{-}$of the arcs (infinite rays) $\mathbb{R}_{+}$and $\mathbb{R}_{-}$of the real circle $\mathbb{R} \times\{0\} \subset \mathbb{C} \times \mathbb{R}=\mathcal{H}$.

Actually, we may assume that the Heisenberg spheres at infinity of the bisectors $\mathfrak{S}$ and $\mathfrak{S}^{\prime}$ have radii 1 and $r_{0}>1$, correspondingly. Then, for a sufficiently small $\epsilon$, 


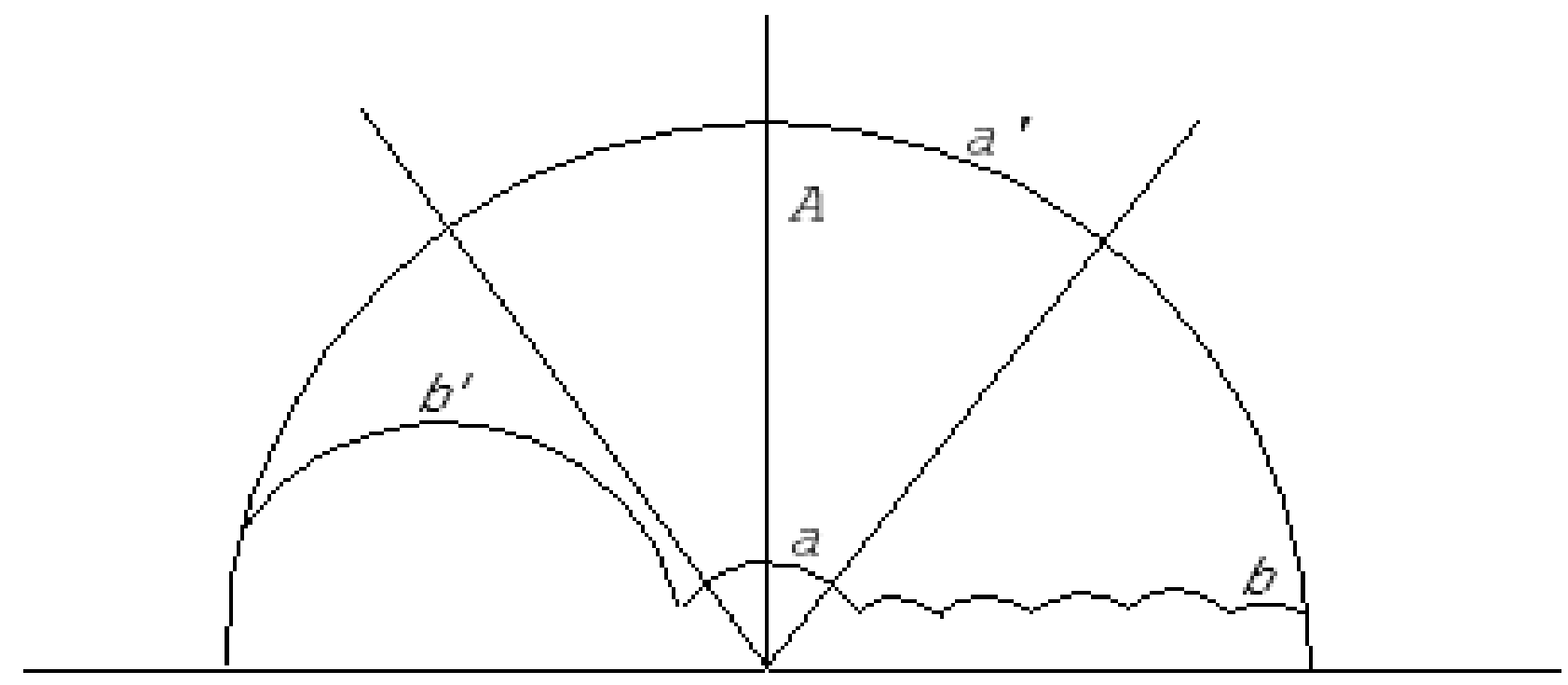

FiguRE 4. $G=G_{1} *_{G_{0}}$.

correspondingly the cones of the $\epsilon$-neighborhoods of the points $(1,0,0),(-1,0,0) \in$ $\mathbb{C} \times \mathbb{R} \times[0,+\infty)$ with respect to the Cygan metric $\rho_{c}$ in $\overline{\mathbb{H}_{\mathbb{C}}^{2}} \backslash\{\infty\}$. Here the Cygan metric $\rho_{c}$ is induced by the following norm (see $[\mathrm{Cy}, \mathrm{Pr}]$ ) in the half-space model $\mathbb{C} \times \mathbb{R} \times[0, \infty)$ of $\overline{\mathbb{H}_{\mathbb{C}}^{2}} \backslash\{\infty\}:$

$$
\|(\xi, v, u)\|_{c}=\left|\|\xi\|^{2}+u-i v\right|^{1 / 2} .
$$

Clearly, we may consider the length $\ell$ of the geodesic $\alpha$ so small that closures of all equidistant halfspaces in $\overline{\mathbb{H}_{\mathbb{C}}^{2}} \backslash\{\infty\}$ bounded by those bisectors and disjoint from the Dirichlet polyhedron $D_{z}(G)$ do not intersect the co-vertical bisector whose infinity is $i \mathbb{R} \times \mathbb{R} \subset \mathbb{C} \times \mathbb{R}$. It follows from the fact (see [G3, Thm VII.4.0.3]) that equidistant half-spaces $\mathfrak{S}_{1}$ and $\mathfrak{S}_{2}$ in $\mathbb{H}_{\mathbb{C}}^{2}$ are disjoint if and only if the intersection half-planes $\mathfrak{S}_{1} \cap \mathbb{H}_{\mathbb{R}}^{2}$ and $\mathfrak{S}_{2} \cap \mathbb{H}_{\mathbb{R}}^{2}$ are disjoint, see Figures 1-4.

Now we are ready to define a quasiconformal bending deformation of the group $G$ along the geodesic $A$, which defines a bending deformation of the complex surface $M=\mathbb{H}_{\mathbb{C}}^{2} / G$ along the given closed geodesic $\alpha \subset S \subset M$.

We specify numbers $\eta$ and $\zeta$ such that $0<\zeta<\pi / 2,0 \leq \eta<\pi-2 \zeta$ and the intersection $C_{+} \cap(\mathbb{C} \times\{0\})$ is contained in the angle $\{z \in \mathbb{C}:|\arg z| \leq \zeta\}$. Then we define a bending homeomorphism $\phi=\phi_{\eta, \zeta}: \mathbb{C} \rightarrow \mathbb{C}$, see Fig. 5 , which bends the real axis $\mathbb{R} \subset \mathbb{C}$ at the origin by the angle $\eta$ :

$$
\phi_{\eta, \zeta}(z)= \begin{cases}z & \text { if }|\arg z| \geq \pi-\zeta \\ z \cdot \exp (i \eta) & \text { if }|\arg z| \leq \zeta \\ z \cdot \exp (i \eta(1-(\arg z-\zeta) /(\pi-2 \zeta))) & \text { if } \zeta<\arg z<\pi-\zeta \\ z \cdot \exp (i \eta(1+(\arg z+\zeta) /(\pi-2 \zeta))) & \text { if } \zeta-\pi<\arg z<-\zeta\end{cases}
$$



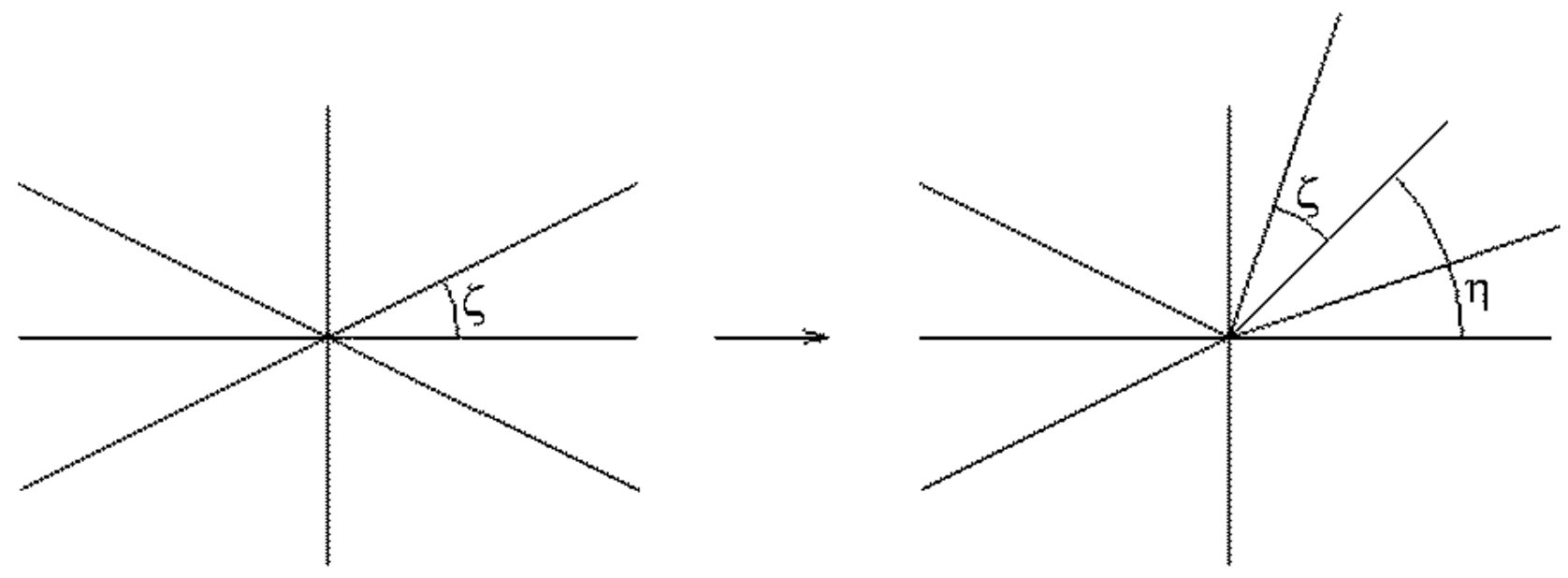

FigURE 5

For negative $\eta, 2 \zeta-\pi<\eta<0$, we set $\phi_{\eta, \zeta}(z)=\overline{\phi_{-\eta, \zeta}(\bar{z})}$. Clearly, $\phi_{\eta, \zeta}$ is quasiconformal with respect to the Cygan norm (4.1) and is an isometry in the $\zeta$-cone neighborhood of the real axis $\mathbb{R}$ because its linear distortion is given by

$$
K\left(\phi_{\eta, \zeta}, z\right)= \begin{cases}1 & \text { if }|\arg z| \geq \pi-\zeta \\ 1 & \text { if }|\arg z| \leq \zeta \\ (\pi-2 \zeta) /(\pi-2 \zeta-\eta) & \text { if } \zeta<\arg z<\pi-\zeta \\ (\pi-2 \zeta+\eta) /(\pi-2 \zeta) & \text { if } \zeta-\pi<\arg z<-\zeta .\end{cases}
$$

Foliating the punctured Heisenberg group $\mathcal{H} \backslash\{0\}$ by Heisenberg spheres $S(0, r)$ of radii $r>0$, we can extend this bending homeomorphism to the whole sphere $S^{3}=\overline{\mathcal{H}}$ at infinity.

Namely, let $W_{+}, W_{-} \subset \mathcal{H}$ be "dihedral angles" with the common vertical axis $\{0\} \times \mathbb{R}$, which are foliated by arcs of real circles connecting points $(0, v)$ and $(0,-v)$ on the vertical axis and intersecting the the $\zeta$-cone neighborhoods of infinite rays $\mathbb{R}_{+}, \mathbb{R}_{-} \subset \mathbb{C}$, correspondingly. For each Heisenberg sphere $S(0, r)$, there is a unique projective ("conformal" with respect to the Cygan metric) transformation $h_{r} \in P U(2,1)$ that maps $S(0, r)$ to the horizontal sphere $(\mathbb{C} \times\{0\}) \cup\{\infty\}$ such that:

(1) It maps the intersection points $S(0, r) \cap(\{0\} \times \mathbb{R})$ to the origin and infinity;

(2) The chain $S(0, r) \cap(\mathbb{C} \times\{0\})$ is pointwise fixed by $h_{r}$;

(3) The intersections $S(0, r) \cap W_{+}$and $S(0, r) \cap W_{+}$are mapped onto the $\zeta$-cone neighborhoods of $\mathbb{R}_{+}, \mathbb{R}_{-} \subset \mathbb{C}$, correspondingly.

Now we define an elementary bending homeomorphism $\varphi=\varphi_{\eta, \zeta}: \mathcal{H} \rightarrow \mathcal{H}$ as the homeomorphism whose restrictions to any Heisenberg sphere $S(0, r)$ are conjugations of the bending $\phi_{\eta, \zeta}$ in the horizontal plane $\mathbb{C} \times\{0\}$ by the projective transformations $h_{r}$, 
Since the transformations $h_{r}$ map chains in $S(0, r)$ (horizontal circles) to chains in the horizontal plane $\mathbb{C} \times\{0\}$ (circles centered at the origin), it directly follows from our construction that the defined homeomorphism $\varphi_{\eta, \zeta}$ preserves the horizontal plane $\mathbb{C} \times\{0\}$, and its restriction to this plane coincides with the bending $\phi_{\eta, \zeta}$. In other words, the elementary bending $\varphi_{\eta, \zeta}$ bends the plane $\mathbb{R} \times \mathbb{R} \subset \mathcal{H}$ along the vertical axis $\{0\} \times \mathbb{R}$, by a given angle $\eta$. Defining $\varphi(\infty)=\infty$ and $\varphi(0)=0$, we naturally extend this homeomorphism to the whole sphere $\overline{\mathcal{H}}$.

It follows from (4.3) that $\varphi_{\eta, \zeta}$ is a $G_{0}$-equivariant quasiconformal homeomorphism in $\overline{\mathcal{H}}$. Moreover, its restrictions to the "dihedral angles" $W_{-}$and $W_{+}$are correspondingly the identity and the unitary rotation $U_{\eta} \in P U(2,1)$ by angle $\eta$ about the vertical axis $\{0\} \times \mathbb{R} \subset \mathcal{H}$.

We can naturally extend the foliation of the punctured Heisenberg group $\mathcal{H} \backslash\{0\}$ by Heisenberg spheres $S(0, r)$ to a foliation of the hyperbolic space $\mathbb{H}_{\mathbb{C}}^{2}$ by bisectors $\mathfrak{S}_{r}$ having those $S(0, r)$ as the spheres at infinity. It is well known (see [M2]) that each bisector $\mathfrak{S}_{r}$ contains a geodesic $\gamma_{r}$ which connects points $\left(0,-r^{2}\right)$ and $\left(0, r^{2}\right)$ of the Heisenberg group $\mathcal{H}$ at infinity, and furthermore $\mathfrak{S}_{r}$ fibers over $\gamma_{r}$ by complex geodesics $Y$ whose circles at infinity are complex circles foliating the sphere $S(0, r)$.

Using those foliations of the hyperbolic space $\mathbb{H}_{\mathbb{C}}^{2}$ and bisectors $\mathfrak{S}_{r}$, we extend the elementary bending homeomorphism $\varphi_{\eta, \zeta}: \overline{\mathcal{H}} \rightarrow \overline{\mathcal{H}}$ at infinity to an elementary bending homeomorphism $\Phi_{\eta, \zeta}: \overline{\mathbb{H}_{\mathbb{C}}^{2}} \rightarrow \overline{\mathbb{H}_{\mathbb{C}}^{2}}$. Namely, the map $\Phi_{\eta, \zeta}$ preserves each of bisectors $\mathfrak{S}_{r}$, each complex geodesic fiber $Y$ in such bisectors, and fixes the intersection points $y$ of those complex geodesic fibers and the complex geodesic connecting the origin and $\infty$ of the Heisenberg group $\mathcal{H}$ at infinity. We complete our extension $\Phi_{\eta, \zeta}$ by defining its restriction to a given (invariant) complex geodesic fiber $Y$ with the fixed point $y \in Y$. This map is obtained by radiating the circle homeomorphism $\left.\varphi_{\eta, \zeta}\right|_{\partial Y}$ to the whole (Poincaré) hyperbolic 2-plane $Y$ along geodesic rays $[y, \infty) \subset Y$, so that it preserves circles in $Y$ centered at $y$ and bends (at $y$, by the angle $\eta$ ) the geodesic in $Y$ connecting the central points of the corresponding arcs of the complex circle $\partial Y$, see Fig.6.
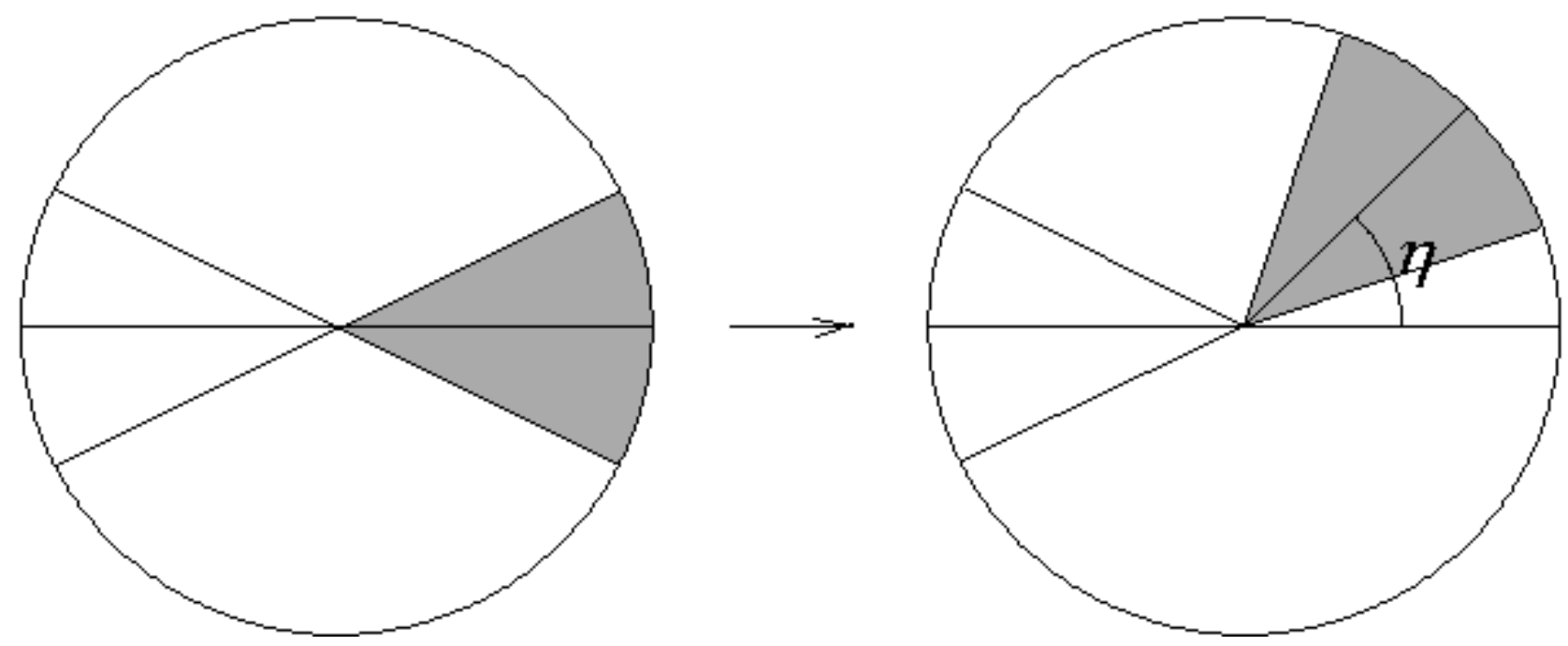
Due to the construction, the elementary bending (quasiconformal) homeomorphism $\Phi_{\eta, \zeta}$ commutes with elements of the cyclic loxodromic group $G_{0} \subset G$. Another most important property of the homeomorphism $\Phi_{\eta, \zeta}$ is the following.

Let $D_{z}(G)$ be the Dirichlet fundamental polyhedron of the group $G$ centered at a given point $z$ on the axis $A$ of the cyclic loxodromic group $G_{0} \subset G$, and $\mathfrak{S}^{+} \subset \mathbb{H}_{\mathbb{C}}^{2}$ be a "half-space" disjoint from $D_{z}(G)$ and bounded by a bisector $\mathfrak{S} \subset \mathbb{H}_{\mathbb{C}}^{2}$ which is different from bisectors $\mathfrak{S}_{r}, r>0$, and contains a side $\mathfrak{s}$ of the polyhedron $D_{z}(G)$. Then there is an open neighborhood $U\left(\overline{\mathfrak{S}^{+}}\right) \subset \overline{\mathbb{H}_{\mathbb{C}}^{2}}$ such that the restriction of the elementary bending homeomorphism $\Phi_{\eta, \zeta}$ to it either is the identity or coincides with the unitary rotation $U_{\eta} \subset P U(2,1)$ by the angle $\eta$ about the "vertical" complex geodesic (containing the vertical axis $\{0\} \times \mathbb{R} \subset \mathcal{H}$ at infinity).

The above properties of quasiconformal homeomorphism $\Phi=\Phi_{\eta, \zeta}$ show that the image $D_{\eta}=\Phi_{\eta, \zeta}\left(D_{z}(G)\right)$ is a polyhedron in $\mathbb{H}_{\mathbb{C}}^{2}$ bounded by bisectors. Furthermore, there is a natural identification of its sides induced by $\Phi_{\eta, \zeta}$. Namely, the pairs of sides preserved by $\Phi$ are identified by the original generators of the group $G_{1} \subset G$. For other sides $\mathfrak{s}_{\eta}$ of $D_{\eta}$, which are images of corresponding sides $\mathfrak{s} \subset D_{z}(G)$ under the unitary rotation $U_{\eta}$, we define side pairings by using the group $G$ decomposition (see Fig. 1-4).

Actually, if $G=G_{1} *_{G_{0}} G_{2}$, we change the original side pairings $g \in G_{2}$ of $D_{z}(G)$-sides to the hyperbolic isometries $U_{\eta} g U_{\eta}^{-1} \in P U(2,1)$. In the case of HNNextension, $G=G_{1} *_{G_{0}}=\left\langle G_{1}, g_{2}\right\rangle$, we change the original side pairing $g_{2} \in G$ of $D_{z}(G)$-sides to the hyperbolic isometry $U_{\eta} g_{2} \in P U(2,1)$. In other words, we define deformed groups $G_{\eta} \subset P U(2,1)$ correspondingly as

$$
G_{\eta}=G_{1} *_{G_{0}} U_{\eta} G_{2} U_{\eta}^{-1} \quad \text { or } \quad G_{\eta}=\left\langle G_{1}, U_{\eta} g_{2}\right\rangle=G_{1} *_{G_{0}} .
$$

We see from (4.5) that the family of representations $G \rightarrow G_{\eta} \subset P U(2,1)$ does not depend on angles $\zeta$ and holomorphically depends on the angle parameter $\eta$. Let us also observe that, for small enough angles $\eta$, the behavior of neighboring polyhedra $g^{\prime}\left(D_{\eta}\right), g^{\prime} \in G_{\eta}$ is the same as of those $g\left(D_{z}(G)\right), g \in G$, around the Dirichlet fundamental polyhedron $D_{z}(G)$. This is because the new polyhedron $D_{\eta} \subset \mathbb{H}_{\mathbb{C}}^{2}$ has isometrically the same (tesselations of) neighborhoods of its side-intersections as $D_{z}(G)$ had. This implies that the polyhedra $g^{\prime}\left(D_{\eta}\right), g^{\prime} \in G_{\eta}$, form a tesselation of $\mathbb{H}_{\mathbb{C}}^{2}$ (with non-overlapping interiors). Hence the deformed group $G_{\eta} \subset P U(2,1)$ is a discrete group, and $D_{\eta}$ is its fundamental polyhedron bounded by bisectors.

Using $G$-compatibility of the restriction of the elementary bending homeomorphism $\Phi=\Phi_{\eta, \zeta}$ to the closure $\overline{D_{z}(G)} \subset \overline{\mathbb{H}_{\mathbb{C}}^{2}}$, we equivariantly extend it from the polyhedron $\overline{D_{z}(G)}$ to the whole space $\mathbb{H}_{\mathbb{C}}^{2} \cup \Omega(G)$ accordingly to the $G$-action. In fact, in terms of the natural isomorphism $\chi: G \rightarrow G_{\eta}$ which is identical on the subgroup $G_{1} \subset G$, we can write the obtained $G$-equivariant homeomorphism $F=F_{\eta}: \overline{\mathbb{H}_{\mathbb{C}}^{2}} \backslash \Lambda(G) \rightarrow \overline{\mathbb{H}_{\mathbb{C}}^{2}} \backslash \Lambda\left(G_{\eta}\right)$ in the following form:

$$
\begin{aligned}
F_{\eta}(x) & =\Phi_{\eta}(x) \quad \text { for } \quad x \in \overline{D_{z}(G)}, \\
F_{\eta} \circ g(x) & =g_{\eta} \circ F_{\eta}(x) \quad \text { for } \quad x \in \overline{\mathbb{H}_{\mathbb{C}}^{2}} \backslash \Lambda(G), g \in G, g_{\eta}=\chi(g) \in G_{\eta} .
\end{aligned}
$$

Due to quasiconformality of $\Phi_{\eta}$, the extended $G$-equivariant homeomorphism $F_{\eta}$ is quasiconformal. Furthermore, its extension by continuity to the limit (real) 
$\Lambda\left(G_{\eta}\right)$ given by the isomorphism Theorem 2.1. Hence we have a $G$-equivariant quasiconformal self-homeomorphism of the whole space $\overline{\mathbb{H}_{\mathbb{C}}^{2}}$, which we denote as before by $F_{\eta}$.

We claim that the family $\left\{F_{\eta}^{*}\right\}$ of representations $F_{\eta}^{*}: G \rightarrow G_{\eta}=F_{\eta} G_{2} F_{\eta}^{-1}$, $\eta \in\left(-\eta_{0}, \eta_{0}\right)$, defines a nontrivial curve $\mathcal{B}:\left(-\eta_{0}, \eta_{0}\right) \rightarrow \mathcal{R}_{0}(G)$ in the variety $\mathcal{R}_{0}(G)$ of faithful discrete representations of $G$ into $P U(2,1)$, which covers a nontrivial curve in the Teichmüller space $\mathcal{T}(G)$ represented by conjugacy classes $[\mathcal{B}(\eta)]=\left[F_{\eta}^{*}\right]$. We call the constructed deformation $\mathcal{B}$ the bending deformation of a given lattice $G \subset P O(2,1) \subset P U(2,1)$ along a bending geodesic $A \subset \mathbb{H}_{\mathbb{C}}^{2}$ with loxodromic stabilizer $G_{0} \subset G$. In terms of manifolds, $\mathcal{B}$ is the bending deformation of a given complex surface $M=\mathbb{H}_{\mathbb{C}}^{2} / G$ homotopy equivalent to its totally real geodesic surface $S_{g} \subset M$, along a given simple geodesic $\alpha$. Summarizing properties of these bending deformations, we have:

Theorem 4.7. Let $G \subset P O(2,1) \subset P U(2,1)$ be a given lattice uniformizing a Riemann 2-surface $S_{p}$ of genus $p \geq 2$. Then, for any simple closed geodesic $\alpha \subset S_{p}=H_{\mathbb{R}}^{2} / G$ and a sufficiently small $\eta_{0}>0$, there is a bending deformation $\mathcal{B}_{\alpha}:\left(-\eta_{0}, \eta_{0}\right) \rightarrow \mathcal{R}_{0}(G)$ of the group $G$ along $\alpha, \mathcal{B}_{\alpha}(\eta)=\rho_{\eta}=F_{\eta}^{*}$, induced by $G$-equivariant quasiconformal homeomorphisms $F_{\eta}: \overline{\mathbb{H}_{\mathbb{C}}^{2}} \rightarrow \overline{\mathbb{H}_{\mathbb{C}}^{2}}$.

Noticing from (4.5) that the bending deformations along disjoint simple closed geodesics are independent, we obtain:

Corollary 4.8. Let $S_{p}=\mathbb{H}_{\mathbb{R}}^{2} / G$ be a closed totally real geodesic surface of genus $p>1$ in a given complex hyperbolic surface $M=\mathbb{H}_{\mathbb{C}}^{2} / G, G \subset P O(2,1) \subset P U(2,1)$. Then there is an embedding $\pi \circ \mathcal{B}: B^{2 p-2} \hookrightarrow \mathcal{T}(M)$ of a real $(2 p-2)$-ball into the Teichmüller space of $M$, defined by bending deformations along disjoint closed geodesics in $M$ and the projection $\pi: \mathcal{D}(M) \rightarrow \mathcal{T}(M)=\mathcal{D}(M) / P U(2,1)$.

Proof. First, we observe that our construction works not only in the case of complex surfaces $M$ with totally real geodesic surface but as well as in the case of a homotopy equivalent surface which has a totally real geodesic piece bounded by closed geodesics. In such a case, the holonomy group of that piece is a non-elementary discrete subgroup in $P U(2,1)$ preserving a totally real geodesic plane $\mathbb{H}_{\mathbb{R}}^{2} \subset \mathbb{H}_{\mathbb{C}}^{2}$. Then we see from (4.5) that bendings along disjoint closed geodesics are independent. In addition to the above construction, we have also to show that our bending deformation is not trivial, and $[\mathcal{B}(\eta)] \neq\left[\mathcal{B}\left(\eta^{\prime}\right)\right]$ for any $\eta \neq \eta^{\prime}$.

The non-triviality of a bending deformation follows directly from (4.5), cf. [A5]. Namely, the restrictions $\left.\rho_{\eta}\right|_{G_{1}}$ of bending representations to a non-elementary subgroup $G_{1} \subset G$ (in general, to a "real" subgroup $G_{r} \subset G$ corresponding to a totally real geodesic piece in the homotopy equivalent surface $S \backsim M$ ) are identical. So if the deformation $\mathcal{B}$ were trivial then it would be conjugation of the group $G$ by projective transformations that commute with the non-trivial real subgroup $G_{r} \subset G$ and pointwise fix the totally real geodesic plane $\mathbb{H}_{\mathbb{R}}^{2}$. This contradicts to the fact that the limit set of any deformed group $G_{\eta}, \eta \neq 0$, does not belong to the real circle containing the Cantor limit set $\Lambda\left(G_{r}\right)$.

The injectivity of the map $\mathcal{B}$ can be obtained by using Élie Cartan $[\mathrm{Ca}]$ angular invariant $\mathbb{A}(x)$ for a triple $x=\left(x^{0}, x^{1}, x^{2}\right)$ of points in $\partial \mathbb{H}_{\mathbb{C}}^{2}$. It satisfies the properties (see [G3]): 
(2) $\mathbb{A}(x)=0$ if and only if $x^{0}, x^{1}$ and $x^{2}$ lie on an $\mathbb{R}$-circle;

(3) $\mathbb{A}(x)= \pm \pi / 2$ if and only if $x^{0}, x^{1}$ and $x^{2}$ lie on a chain ( $\mathbb{C}$-circle);

(4) For two triples $x$ and $y, \mathbb{A}(x)=\mathbb{A}(y)$ if and only if there exists $g \in P U(2,1)$ such that $y=g(x)$; furthermore, such a $g$ is unique provided that $\mathbb{A}(x)$ is neither zero nor $\pm \pi / 2$.

(5) $\mathbb{A}(x)=-\mathbb{A}(y)$ if and only if there exists an antiholomorphic automorphism $g \in \operatorname{Isom} \mathbb{H}_{\mathbb{C}}^{2}$ such that $y=g(x)$.

Namely, let $g_{2} \in G \backslash G_{1}$ be a generator of the group $G$ in (4.5) whose fixed point $x^{2} \in \Lambda(G)$ lies in $\mathbb{R}_{+} \times\{0\} \subset \mathcal{H}$, and $x_{\eta}^{2} \in \Lambda\left(G_{\eta}\right)$ the corresponding fixed point of the element $\chi_{\eta}\left(g_{2}\right) \in G_{\eta}$ under the free-product isomorphism $\chi_{\eta}: G \rightarrow G_{\eta}$. Due to our construction, one can see that the orbit $\gamma\left(x_{\eta}^{2}\right), \gamma \in G_{0}$, under the loxodromic (dilation) subgroup $G_{0} \subset G \cap G_{\eta}$ approximates the origin along a ray $(0, \infty)$ which has a non-zero angle $\eta$ with the ray $\mathbb{R}_{-} \times\{0\} \subset \mathcal{H}$. The latter ray also contains an orbit $\gamma\left(x^{1}\right), \gamma \in G_{0}$, of a limit point $x^{1}$ of $G_{1}$ which approximates the origin from the other side. Taking triples $x=\left(x^{1}, 0, x^{2}\right)$ and $x_{\eta}=\left(x^{1}, 0, x_{\eta}^{2}\right)$ of points which lie correspondingly in the limit sets $\Lambda(G)$ and $\Lambda\left(G_{\eta}\right)$, we have that $\mathbb{A}(x)=0$ and $\mathbb{A}\left(x_{\eta}\right) \neq 0, \pm \pi / 2$. Due to Theorem 2.1 , both limit sets are topological circles which however cannot be equivalent under a hyperbolic isometry because of different Cartan invariants (and hence, again, our deformation is not trivial).

Similarly, for two different values $\eta$ and $\eta^{\prime}$, we have triples $x_{\eta}$ and $x_{\eta^{\prime}}$ with different (non-trivial) Cartan angular invariants $\mathbb{A}\left(x_{\eta}\right) \neq \mathbb{A}\left(x_{\eta^{\prime}}\right)$. Hence two topological circles $\Lambda\left(G_{\eta}\right)$ and $\Lambda\left(G_{\eta^{\prime}}\right)$ are not hyperbolically isometric. This completes the proof of injectivity and of the whole Corollary.

One can apply the above proof to a general situation of bending deformations of a complex hyperbolic surface $M=\mathbb{H}_{\mathbb{C}}^{2} / G$ whose holonomy group $G \subset P U(2,1)$ has a non-elementary subgroup $G_{r}$ preserving a totally real geodesic plane $\mathbb{H}_{\mathbb{R}}^{2}$. In other words, such a complex surfaces $M$ has an embedded totally real geodesic surface with geodesic boundary. In particular all complex surfaces constructed in [GKL] with a given Toledo invariant lie in this class. So we immediately have:

Corollary 4.9. Let $M=\mathbb{H}_{\mathbb{C}}^{2} / G$ be a complex hyperbolic surface with embedded totally real geodesic surface $S_{r} \subset M$ with geodesic boundary, and $\mathcal{B}:(-\eta, \eta) \rightarrow$ $\mathcal{D}(M)$ a bending deformation of $M$ along a simple closed geodesic $\alpha \subset S_{r}$. Then the map $\pi \circ \mathcal{B}:(-\eta, \eta) \rightarrow \mathcal{T}(M)=\mathcal{D}(M) / P U(2,1)$ is a smooth embedding provided that the limit set $\Lambda(G)$ of the holonomy group $G$ does not belong to the $G$-orbit of the real circle $S_{\mathbb{R}}^{1}$ and the chain $S_{\mathbb{C}}^{1}$, where the latter is the infinity of the complex geodesic containing a lift $\tilde{\alpha} \subset \mathbb{H}_{\mathbb{C}}^{2}$ of the closed geodesic $\alpha$, and the former one contains the limit set of the holonomy group $G_{r} \subset G$ of the geodesic surface $S_{r}$.

Remark 4.10. It follows from the above construction of the bending homeomorphism $F_{\eta, \zeta}$, that the deformed complex hyperbolic surface $M_{\eta}=\mathbb{H}_{\mathbb{C}}^{2} / G_{\eta}$ fibers over the pleated hyperbolic surface $S_{\eta}=F_{\eta}\left(\mathbb{H}_{\mathbb{R}}^{2}\right) / G_{\eta}$ (with the closed geodesic $\alpha$ as the singular locus). The fibers of this fibration are "singular real planes" obtained from totally real geodesic 2-planes by bending them by angle $\eta$ along complete real geodesics. These (singular) real geodesics are the intersections of the complex geodesic connecting the axis $A$ of the cyclic group $G_{0} \subset G$ and the totally real geodesic planes that represent fibers of the original fibration in $M=\mathbb{H}_{\mathbb{C}}^{2} / G$. 


\section{REFERENCES}

[A1] Boris Apanasov, Discrete groups in Space and Uniformization Problems. - Math. and Appl., 40, Kluwer Academic Publishers, Dordrecht, 1991.

[A2] _ Nontriviality of Teichmüller space for Kleinian group in space.- Riemann Surfaces and Related Topics: Proc. 1978 Stony Brook Conference (I.Kra and B.Maskit, eds), Ann. of Math. Studies 97, Princeton Univ. Press, 1981, 21-31.

[A3] Thurston's bends and geometric deformations of conformal structures.- Complex Analysis and Applications'85, Publ. Bulgarian Acad. Sci., Sofia, 1986, 14-28.

[A4] Deformations of conformal structures on hyperbolic manifolds.- J. Diff. Geom. 35 (1992), 1-20.

[A5] Conformal geometry of discrete groups and manifolds. - W. de Gruyter, Berlin- New York, to appear.

[A6] Canonical homeomorphisms in Heisenberg groups induced by isomorphisms of discrete subgroups of $P U(n, 1)$. - Russian Acad. Sci. Dokl. Math., to appear.

[A7] _ Quasiconformality and geometrical finiteness in Carnot-Carathéodory and negatively curved spaces. - Math. Sci. Res. Inst. at Berkeley, 1996-019.

[ACG] Boris Apanasov, Mario Carneiro and Nikolay Gusevskii, Some deformations of complex hyperbolic surfaces. - In preparation.

[AG] Boris Apanasov and Nikolay Gusevskii, The boundary of Teichmüller space of complex hyperbolic surfaces. - In preparation.

[AX1] Boris Apanasov and Xiangdong Xie, Geometrically finite complex hyperbolic manifolds.Preprint, 1995.

[AX2] _ Manifolds of negative curvature and nilpotent groups.- Preprint, 1995.

[Be] A.F. Beardon, The geometry of discrete groups.- Springer-Verlag, Berlin/New York, 1983.

[Bu] Peter Buser, The collar theorem and examples.- Manuscr. Math. 25 (1978), 349-357.

[BS] D. Burns and S. Shnider, Spherical hypersurfaces in complex manifolds. - Inv. Math., 33(1976), 223-246.

[CG] S. Chen and L. Greenberg, Hyperbolic spaces.- Contributions to Analysis, Academic Press, New York, 1974, 49-87.

[Ca] Élie Cartan, Sur le groupe de la géométrie hypersphérique.- Comm. Math. Helv. 4 (1932), 158-171.

[C1] Kevin Corlette, Archimedian superrigidity and hyperbolic geometry. - Ann. of Math. 135 (1992), 165-182.

[Cy] J. Cygan, Wiener's test for Brownian motion on the Heisenberg group. - Colloquium Math. 39(1978), 367-373.

[FG] Elisha Falbel and Nikolay Gusevskii, Spherical CR-manifolds of dimension 3.- Bol. Soc.Bras.Mat. 25(1994), 31-56

[G1] William Goldman, Representations of fundamental groups of surfaces.- Geometry and topology (J.Alexander and J.Harer, Eds), Lect. Notes Math. 1167, Springer, 1985, 95117.

[G2] _ Geometric structures on manifolds and varieties of representations. - Geometry of Group Representations (A.Magid and W. Goldman, Eds), Contemp. Math. 74 (1988), 169-198.

[G3] Complex hyperbolic geometry. - Oxford Univ. Press, to appear.

[GKL] W.Goldman, M.Kapovich and B.Leeb, Complex hyperbolic manifolds homotopy equivalent to a Riemann surface. - Preprint, 1995.

[GM] William Goldman and John Millson, Local rigidity of discrete groups acting on complex hyperbolic space.- Invent. Math. 88(1987), 495-520.

[GP] William Goldman and John Parker, Complex hyperbolic ideal triangle groups.- J. reine angew. Math. 425 (1992), 71-86.

[Gu] Nikolay Gusevskii, Colloquium talk, Univ. of Oklahoma, Norman, December 1995.

[JM] Dennis Johnson and John Millson, Deformation spaces associated to compact hyperbolic manifolds.- Discrete Groups in Geometry and Analysis: Papers in Honor of G.D. Mostow on His Sixtieth Birthday, Ed. R. Howe, Birkhauser, Boston, 1987, 48-106.

[Ke] Linda Keen, Collars on Riemann surfaces.- Discontinuous groups and Riemann surfaces, 
[KR] Adam Koranyi and Martin Reimann, Quasiconformal mappings on the Heisenberg group. - Invent. Math. 80 (1985), 309-338.

[Ko] Christos Kourouniotis, Deformations of hyperbolic structures.- Math. Proc. Cambr. Phil. Soc. 98 (1985), 247-261.

[Mas] Bernard Maskit, Kleinian groups. - Springer-Verlag, 1987.

[Ma] Georgii A. Margulis, Discrete groups of motions of manifolds of non-positive curvature.Proc. ICM, Vancouver, v.2, 1974, 21-34 (Russian); Engl. Transl.: Amer. Math. Soc. Translations 109 (1977), 33-45.

[M1] George D. Mostow, Strong rigidity of locally symmetric spaces.- Princeton Univ. Press, 1973.

[M2] _ On a remarkable class of polyhedra in complex hyperbolic space. - Pacific J. Math., 86(1980), 171-276.

[P] Pierre Pansu, Métriques de Carnot-Carathéodory et quasiisométries des espaces symmétries de rang un.- Ann. Math. 129 (1989), 1-60.

[Pr] John Parker, Shimizu's lemma for complex hyperbolic space. - Intern. J. Math. 3:2 (1992), 291-308.

[Th] William Thurston, The geometry and topology of three-manifolds. - Lect. Notes, Princeton Univ., 1981.

[To] Domingo Toledo, Representations of surface groups on complex hyperbolic space.- J. Diff. Geom. 29 (1989), 125-133.

[Tu] Pekka Tukia, On isomorphisms of geometrically finite Kleinian groups.- Publ. Math. IHES 61(1985), 171-214.

[V] Serguei K. Vodopyanov, Quasiconformal mappings on Carnot groups.- Russian Dokl. Math., to appear

[Ya] S.T.Yau, Calabi's conjecture and some new results in algebraic geometry.- Proc. Nat. Acad. Sci 74 (1977), 1798-1799.

[Yu] Chengbo Yue, Dimension and rigidity of quasi-Fuchsian representations.- Ann. of Math. 143 (1996)331-355.

Dept. of Math., Univ. of Oklahoma, Norman, OK 73019

Mathematical Sciences Research Institute, Berkeley, CA 94720-5070

E-mail address: apanasov@uoknor.edu 